\title{
Der Wert Europas und seine Grenzen: die EU als außenpolitische Gestaltungsmacht mit Defiziten
}

\author{
Ulrike Guérot*
}

Eine realistische Bewertung der aktuellen Lage der europäischen Außenpolitik muss zu dem Schluss kommen, dass Europa mehr denn je von einer geeinten und effizienten europäischen Außenpolitik entfernt ist: Libyen, Syrien, Nord Stream-Pipeline oder Kosovo sind hier nur Reizworte. Die Neueinrichtung des Europäischen Auswärtigen Dienstes hat daran bisher nur wenig geändert, auch wenn in anderen außenpolitischen Politikbereichen, zum Beispiel einer gemeinsamen EU-Außenpolitik mit Blick auf Russland, durchaus Fortschritte erreicht wurden. Catherine Ashton, die Hohe Vertreterin der Union für Außen- und Sicherheitspolitik, hat sich trotz ,Doppelhut ‘ und einer eindeutigen Stärkung der europäischen Außenpolitik (Gemeinsame Außen- und Sicherheitspolitik, GASP/Gemeinsame Sicherheitsund Verteidigungspolitik, GSVP) durch den Vertrag von Lissabon noch nicht als Gesicht oder einheitliche Stimme europäischer Außenpolitik behaupten können und dies hat nicht nur persönliche, sondern auch institutionelle Gründe. Jenseits vom oft beklagten mangelnden ,politischen Willen' - vor allem der größeren EU-Mitgliedstaaten, die überhaupt geltend machen können, über eine eigenständige Außenpolitik zu verfügen - sollen im Folgenden vier Thesen beziehungsweise Ursachen diskutiert werden, die die Entwicklung einer schlagkräftigen, einheitlichen, kohärenten und effizienten europäischen Außenpolitik zumindest erschweren.

- Die Erosion von Außenpolitik im Allgemeinen - und die Auswirkungen auf die Europäische Union und ihre angestrebte Außenpolitik

- Werte für die Europäische Union - Interessen für die Nationalstaaten? Die EU-Außenpolitik zwischen Anspruch und Wirklichkeit

- Wann ist nationale Interessenvertretung Provinzialismus? Anmerkungen zur strukturellen außenpolitischen Unfähigkeit europäischer Staaten

- Und wenn Europa nur ein Mythos war? Welche Chancen hat eine pragmatische EU-Außenpolitik und worin könnte sie bestehen?

\section{Die Erosion der Bedeutung von Außenpolitik im Allgemeinen - und die Auswirkungen auf die Europäische Union und ihre angestrebte Außenpolitik}

Die erste These lautet, dass der ,Primat der Politik“ im außenpolitischen Bereich ebenso verloren gegangen ist wie im innenpolitischen beziehungsweise wirtschaftspolitischen Bereich. Vielleicht kann man sogar behaupten, dass im ,europäischen Westen' der innen- wie außenpolitische Primat der Politik überhaupt nur durch die strukturelle Zementierung der wirtschaftlichen Rahmenbedingungen durch den Kalten Krieg, die Übernahme der Verteidigungskosten durch die USA und den fehlenden Druck der Globalisierung möglich war.

* Dr. Ulrike Guérot, Representative for Germany and Senior Policy Fellow des European Council on Foreign Relations (ECFR) Berlin.

Der Artikel basiert auf einem Vortrag für den Workshop „Der Wert Europas“ am 16. Januar 2012 im Auswärtigen Amt, Berlin. 
Die Zeit von 1949 bis 1989 war damit eher die ,Ausnahme` denn die Regel für eine deutsche oder europäische Außenpolitik oder anders formuliert: Außenpolitik war ein strategischer Luxus im wirtschaftlich geschützten Raum, indem eine normativ gebundene Interessenspolitik noch gelingen konnte. Ebenso wie derzeit im binnenpolitischen Raum das Wegbrechen des ,öffentlichen Raumes' (soziale Kohäsion, Bildung, Solidarität, etc.) ${ }^{1}$ zu beobachten ist, ist im internationalen Raum ein Wegbrechen der ,common goods' beziehungsweise der in-

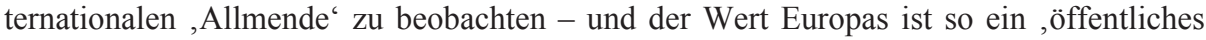
Gut $^{6}{ }^{2}$ Eine wertegebundene Außenpolitik, die sich streng an immateriellen Werten (Menschenrechte, ,good governance', ,rule of law', Solidarität, etc.) orientiert - wenn es sie denn je gegeben hat - und die auf einem strategischen Konzept der Entwicklung von ,Weltinnenpolitik' beruht oder beruhen würde, ist nicht zu erkennen beziehungsweise wird von der Europäischen Union immer weniger politisch unterfüttert, wirtschaftlich finanziert oder militärisch gesichert. Im Spannungsfeld zwischen internationaler Solidarität und Selbstbehauptung ist die Europäische Union mithin zu wertegeleiteter, immaterieller Solidarität immer weniger bereit und zur interessengeleiteten Selbstbehauptung immer weniger in der Lage: ${ }^{3}$ Ersteres, weil ihr die ökonomischen Ressourcen und letztlich die politische Bereitschaft für eine neue globale Wohlstandsverteilung fehlen; Letzteres, weil ihr die militärischen Mittel zur Sanktionsfähigkeit von Machtansprüchen fehlen.

Die tendenzielle Abkehr von kodifiziertem, internationalem Recht, also die strukturelle Rückkehr von ,Macht vor Recht ‘ beziehungsweise die, Rückkehr der Geschichte ${ }^{4}$ und damit eine Rückorientierung zu souveränistischen Konzepten von Außenpolitik in Kontrast zur multilateralen Orientierung und zu Prinzipien wie Rechtsstaatlichkeit, ,rule of law“ oder ,good governance‘ ist derzeit ein weltweites Phänomen. Es trifft die Europäische Union aber ganz besonders, da sie als Spezifikum - im Gegensatz zu anderen Mächten wie China oder Russland - eben über keine oder nicht ausreichende Elemente von Staatlichkeit verfügt, die es ihr ermöglichen würden, in einem neuen ,Konzert der Mächte' beziehungsweise einem ,Konzert der neuen Mächte‘ dezidierten globalen Einfluss zu gewinnen oder ihr eigenes Wertesystem durchzusetzen. Dies gilt zum Beispiel mit Blick auf ihre demokratietheoretischen Vorstellungen oder auch mit Blick auf ihre sozialpolitischen Maximen. Außenpolitik wird damit de facto zunehmend durch bloße Handelspolitik ersetzt und mehr oder weniger ausschließlich in die - strategielose - Matrix des wirtschaftlichen Nutzens eines Staates gestellt.

Dazu kommt, dass Staaten de facto nicht mehr die alleinigen und oft auch nicht mehr die zentralen Akteure außenpolitischen Handelns sind, sondern zum Beispiel internationale Nicht-Regierungs-Organisationen, internationale Konzerne oder neuerdings (siehe Somalia) wieder Piraten zu schlagkräftigen Akteuren im internationalen System geworden sind. Probleme wie etwa Landraub (Land-Grabbing) in Afrika zum Zweck des Agraranbaus, etwa durch große Nahrungsmittelkonzerne ${ }^{5}$ sind genau auf die Erosion staatlicher Macht zurückzuführen - und die zunehmende Unfähigkeit der internationalen Staatengemeinschaft, mit ,failing states‘ umzugehen beziehungsweise dieses Machtvakuum zu kompensieren.

1 Dazu wegweisend Richard Sennett: Verfall und Ende des öffentlichen Lebens. Die Tyrannei der Intimität, Berlin 2008.

2 Dazu Roland Tichy/Ulrike Guérot: Vom eigenen Garten zur weltweiten Ressourcenverteilung, in: Aus Politik und Zeitgeschichte 28-30/2011, S. 15-21.

3 Siehe hier Claudio Franzius/Ulrich K. Preuß: Solidarität und Stärke - Zur Zukunft der Europäischen Union, herausgegeben von der Heinrich-Böll-Stiftung, Berlin 2011.

4 Ganz im Gegensatz zu Francis Fukuyama: Das Ende der Geschichte. Wo stehen wir?, 4. Auflage, Reinbek 1992.

5 Vgl. zum Beispiel Sarah Steffen: UN approves guidelines against land grabbing, in: dw.de, 12.5.2012. 
Dies bedeutet gleichsam eine Ver-Mittelalterlichung von Außenpolitik: gemeint ist, dass etwa Handelsgesellschaften zu großen außenpolitischen Akteuren werden, die gleichsam staatliche oder gar hoheitliche Aufgaben übernehmen. ${ }^{6}$ Das multilaterale System - siehe zum Beispiel die Welthandelsorganisation, die $\mathrm{NATO}^{7}$ oder der Treaty of Non-Proliferation ${ }^{8}$ ist eher in Erosion, denn im Aufbau begriffen. Die Europäische Union verliert derzeit unter anderem ihre normative Rechtsetzungsfähigkeit in den Vereinten Nationen ${ }^{9}$ sowie ihre Koalitionsfähigkeit.

\section{Werte für die Europäische Union - Interessen für die Nationalstaaten? Die EU- Außenpolitik zwischen Anspruch und Wirklichkeit}

Die zweite These lautet, dass die Europäische Union, blickt man auf das, was bisher vertraglich kodifiziert ist, durchweg die Verfolgung von immateriellen Werten in ihrer Außenpolitik verspricht, die auf nationaler Ebene aber oft nicht eingelöst werden können (zum Beispiel die Aufnahme von Flüchtlingen in Lampedusa etc.). Kurz gesagt: Die EU-Außenpolitik verspricht zu viel. Im Grunde sind die Europäische Union und ihre Mitgliedstaaten , arbeitsteilig' aktiv - die Union ist ,good cop' die Mitgliedstaaten sind ,bad cop'. Die Europäische Union proklamiert zum Beispiel nachhaltige Klimapolitik, eine Finanztransaktions- oder $\mathrm{CO}_{2}$-Steuer, Flüchtlingsaufnahme oder materielle Unterstützung der ,Arabellion“, doch die Nationalstaaten haben politische Bedenken ob der Kosten. Die EU-Außenpolitik ist damit oft gleichsam der Fluchtpunkt oder das Feigenblatt für die guten deklaratorischen Absichten, während die EU-Mitgliedstaaten realpolitisch geerdet sind - und die EU-Außenpolitik steht damit allzu oft zwischen Anspruch und Wirklichkeit.

Zudem ist die Sollbruchstelle der demokratischen Legitimität der EU-Außenpolitik besonders groß: Die ,G-2/8/20` Welt bedeutet de facto eine ,Präsidialisierung` oder ,Exekutivierung'von Außenpolitik, der noch kein wirkliches Pendant beziehungsweise irgendeine Form parlamentarischer Kontrolle/Verantwortlichkeit auf europäischer Ebene gegenübersteht - ein Vakuum, das auch das Europäische Parlament nur sehr begrenzt füllen kann. Mit Jürgen Habermas gesprochen ${ }^{10}$ steht die Europäische Union vor der Entscheidung zwischen transnationaler Demokratie (auch in der Außenpolitik) und post-demokratischem Exekutivföderalismus - und scheint Letzteres zu wählen. Zudem ist der Parlamentsvorbehalt bei militärischen Entscheidungen zum Beispiel in Deutschland strukturell mit Blick auf eine potenzielle strategische Machtprojektion der Europäischen Union ein großes Problem, da er die Sanktionsfähigkeit der Europäischen Union in entscheidenden, nämlich unter anderem den militärischen Bereichen der GASP beziehungsweise GSVP untergräbt.

Ein Teil dieser institutionellen Probleme ist durch den Vertrag von Lissabon hausgemacht: Die nationalen Außenminister haben durch ihn allgemein an Bedeutung im EU-System verloren. Gleichzeitig zeigt sich, dass jenseits der vertraglichen EU-Strukturen die Machtbasis für eine glaubwürdige EU-Außenpolitik eben nicht gegeben ist, wenn sich zum Beispiel die ,big three' nicht einigen können (als Beispiel siehe Libyen!).

6 So eine der zentralen Thesen von Parag Khannan: When cities rule the world, in: McKinsey \& Company: What Matters, 7.1.2011, abrufbar unter: http://whatmatters.mckinseydigital.com/cities/when-cities-rule-the-world (letzter Zugriff: 28.3.2012).

7 North Atlantic Treaty Organization.

8 Vertrag über die Nicht-Verbreitung von Nuklearwaffen.

9 Siehe dazu Franziska Brantner/Richard Gowan: A Global Force for Human Rights? An audit of European Power at the UN, ECFR Policy Paper, 2008.

10 Vgl. Jürgen Habermas: Zur Verfassung Europas, Frankfurt Main 2011. 
Interessanterweise ist mit jedem Ausbau der GASP/GSVP die Rolle der kleinen EU-Mitgliedstaaten in der EU-Außenpolitik gesunken, während die großen Mitgliedstaaten, insbesondere Deutschland, einen Machtzuwachs erlebt haben und weite Teile europäischer Außenpolitik, zum Beispiel jene mit Blick auf China oder Russland, von Deutschland allein durch seine ökonomische Dominanz de facto determiniert werden. EU-Außenpolitik ist damit oft genau dann Makulatur, wenn (vermeintlich), deutsche Interessen ' einer gemeinsamen EU-Außenpolitik entgegenstehen. Dies gilt graduell unterschiedlich zum Beispiel für die Beziehungen der Europäischen Union zu China oder Russland und die Libyen-Entscheidung. Diese strukturelle Problematik ist natürlich auch nicht auf Deutschland beschränkt, sondern lässt sich analog beispielsweise für die Haltung Italiens mit Blick auf Libyen oder Syrien feststellen (oder in anderen Sachverhalten für Frankreich oder Großbritannien). Dabei ist indes zu bedenken, dass die deutsche Haltung in anderer Gewichtung richtungsweisend für die Europäische Union ist. Die wieder neue und letztlich noch ungelöste Frage einer deutschen Hegemonie in Europa ${ }^{11}$ entwickelt sich derzeit zum größten Hemmschuh einer europäischen Außenpolitik. Die Crux hierbei dürfte es sein, die ökonomische Macht Deutschlands - als Antithese zum Mythos einer , deutschen Schweiz - einer politischen Macht Europas zuzuführen oder die ökonomische Schwerkraft Deutschlands gewinnbringend als Resonanzboden für eine wertegebundene Interessenpolitik für ganz Europa zu nutzen. Kurz: Es liegt essenziell an Deutschland, ob die Europäische Union im 21. Jahrhundert zu einer verantwortungsvollen, internationalen Gestaltungsmacht wird und ob sich Deutschland in eine EU-Außenpolitik nicht nur einbinden lässt, sondern eben diese auch aktiv gestalten wird; oder ob Deutschland versucht, aus der Europäischen Union herauszuwachsen und allein eine globale Rolle anzustreben.

\section{Wann ist nationale Interessenvertretung Provinzialismus? Anmerkungen zur strukturellen außenpolitischen Unfähigkeit europäischer Staaten}

Die dritte These lautet, dass das ,Globalisierungsparadox ${ }^{612}$ dazu führt, dass Außenpolitik nicht mehr ohne, das Volk' gemacht werden kann, ,das Volk‘ aber keine Außenpolitik mehr will. Im Unterschied zur Zeit zwischen 1949 und 1989, wo alle Außenpolitik dem Paradigma der nationalen Sicherheit und damit der absoluten Bedrohung unterlag - und damit in allen europäischen Staaten, West wie Ost, nationale außenpolitische Konsense hervorbringen konnte - treibt heute die Innenpolitik oder genauer gesagt der populistische Druck die europapolitische und außenpolitische Diskussion. In dieser Bewegung weg von Geo-Strategie hin zu Geo-Ökonomie können immaterielle, geo-strategische Vorteile einer EU-Außenpolitik nicht mehr oder zumindest immer weniger überzeugen, wenn oder zumal sie mit wirtschaftlichen Verlusten assoziiert werden. Außenpolitische Vorteile - zum Beispiel Frieden, Stabilität, Sicherheit oder energiepolitische Unabhängigkeit - sind zudem abstrakt und stehen auf einer anderen Zeitlinie, sie sind also weniger unmittelbar sichtbar und kontrastieren daher oftmals mit unmittelbaren Wählerinteressen oder auch teilweise populistischen Angstszenarien (Furcht vor Migration, Terror, etc.).

Abstrakte, kollektive Langzeitziele und öffentliche Güter können in Demokratien, die unmittelbaren und kurzfristigen Wahlzwängen- beziehungsweise Wahlerfolgen unterliegen, oft nur mühsam geltend gemacht werden. In allen europäischen Mitgliedstaaten schrumpfen

11 Vgl. hier weiterführend Christoph Schöneberger: Hegemon wider Willen. Zur Stellung Deutschlands in der Europäischen Union, in: Merkur 1/2012.

12 Dani Rodnik: Das Globalisierungsparadox. Die Demokratie und die Zukunft der Weltwirtschaft, München 2011. 
so die internationalen politischen Eliten, die angesichts einer wachsenden populistischen Herausforderung durch die Globalisierungsverlierer, die in einigen Mitgliedstaaten längst einen Anteil von 30 Prozent der Bevölkerung erreicht haben, eine europäische Öffnung ihres Staates noch verteidigen. Der aktuelle Populismus in EU-Europa (zum Beispiel aktuell auch die Forderungen, das Schengen-Abkommen einzuschränken) ist nicht von ungefähr fast immer die Kombination von Anti-Europa- und Anti-Migration-Diskursen. Die relative Verarmung der europäischen Arbeiterklasse und das Auseinanderdriften der Gewinne einer politisch fast abgekoppelten europäischen Exportindustrie von politischer Wohlstandsredistribution - national wie europäisch - sind eine nicht zu unterschätzende Gefahr für das gesellschaftliche Gefüge und damit auch eine auf Öffnung bedachte zukünftige europäische Außenpolitik. Die Debatte über eine zukünftige EU-Außenpolitik steht damit vor dem immanenten Risiko, gleichsam von irrationalen Argumenten weggefegt zu werden: so war zum Beispiel zeitweise selbst der Verweis auf den wirtschaftlichen Gewinn Deutschlands durch den europäischen Binnenmarkt in der Hochphase der Eurokrise nur mit Mühe und Not letztlich ein valides Argument, um die Deutschen zum Beispiel von der Notwendigkeit eines Euro-Rettungsschirms zu überzeugen, da ein Zerfall des Euro möglicherweise auch die Existenz des Binnenmarktes bedroht hätte. Zudem liegt es in der Natur der Sache, dass der immaterielle außenpolitische Wert Europas (Friede, Sicherheit) in der Diskussion zahlenmäßig nicht richtig quantifiziert werden kann. Damit ist das politische Argument über den vermeintlichen Verlust ebendieser Werte immer zahnlos.

Hinzu kommt, dass die Europäische Union und damit auch eine EU-Außenpolitik zum Opfer ihrer eigenen institutionellen Komplexität geworden ist und die europäische Integration an sich, bedingt durch sowohl den innereuropäischen gesellschaftlichen Wandel als auch die globalen Veränderungen, immer weniger gesellschaftliche Träger und Fürsprecher hat. Eine neue europäische ,Erzählung', die auch in Zeiten von Geo-Ökonomie und Globalisierung - beides Herausforderungen, die an das nationale Schutzbedürfnis appellieren - eine europäische Außenpolitik und damit ein gemeinsames Agieren, eine konsequente Agenda der Öffnung, der Verantwortung und des internationalen Engagements für die Europäische Union begründet, muss noch gefunden werden. Die Klammer der Globalisierung scheint für eine solche ,Erzählung‘ nicht unbedingt zu reichen, solange nicht klar ist, ob Europa Schutzraum oder im Gegenteil Sprungbrett für die Globalisierung sein soll und es politisch austariert ist, wer von den EU-Bürgern durch die Globalisierung in welchem Umfang profitiert. Diese Frage umfasst auch das neue, inner-europäische Spannungsverhältnis und das Risiko einer gleichsam kulturellen Spaltung innerhalb der Europäischen Union zwischen Kreditgeberländern und Kreditnehmerländern im Zuge der Euro-Krise beziehungsweise die Frage, ob es der Europäischen Union mittelfristig gelingt, wieder an frühere, gesamteuropäische Ziele der wirtschaftlichen und sozialen Kohäsion anzuknüpfen. Die Europäische Union steht vor der komplexen Aufgabe, gesamteuropäisch ihren ,contrat social' neu zu verhandeln, und zwar sowohl das Verhältnis zwischen Markt und Staat als auch jenes zwischen Kapital und Arbeit.

Die Kontextualisierung von außenpolitischen Problemen heißt auch die Verknüpfung von innen- und außenpolitischen Problemen und damit auch die Wertschätzung von immateriellen, außenpolitischen Errungenschaften (Friede, Stabilität). Dies gelingt indes immer weniger in der Kommunikation über Europa. Damit gelingt ebenso wenig die Verknüpfung von außenpolitischen Optionen oder Lösungen (zum Beispiel EU-Osterweiterung oder Aufnahme der Türkei) mit innenpolitischen Problemen (zum Beispiel Arbeitskräftemangel oder Energie-/Pipelinesicherheit). Anders formuliert: Oft proklamiert die Europäische Union eine 
,Agenda der Öffnung‘, die europäischen Mitgliedstaaten indes forcieren zunehmend eine ,Agenda der Abschottung' und koppeln damit letztlich mögliche europäische Lösungen von nationalstaatlichen Problemen ab.

Die europäische Außenpolitik wird derzeit zerrieben im strategischen Vakuum Europas nach dem Ende der Einbettung Europas in den doppelten Konsens der einstigen Jalta- und Maastricht-Ordnung.

\section{Und wenn Europa nur ein Mythos war? Welche Chancen hat eine pragmatische EU- Außenpolitik und worin könnte sie bestehen?}

Die vierte These schließlich lautet, dass eine effektive und gemeinsame europäische Außenpolitik de facto einen politisch und fiskalisch noch weiter integrierten Euro zur Grundlage haben müsste. Fakt ist, dass starke Kräfte in verschiedenen EU-Mitgliedstaaten, darunter auch in Deutschland, krisenbedingt und durch die Aufkündigung der Geschichte als Klammer und Begründung für die europäische Integration angefangen haben, den Nutzen Europas allgemein infrage zu stellen. ${ }^{13}$ Sowohl in Deutschland, Frankreich und Großbritannien und natürlich auch in verschiedenen kleineren EU-Mitgliedstaaten werden nationale außenpolitische Optionen verstärkt diskutiert - obgleich sich in Deutschland seit einigen Wochen gerade wieder eine zaghafte Trendwende und damit eine erneute Hinwendung zu einem ,mehr-Europa ${ }^{6}$-Argument abzeichnet ${ }^{14}$ - nachdem der europapolitische Konsens des Landes im letzten Jahr zeitweilig vom Entgleisen bedroht war.

Die Abkehr vom Ziel einer immer engeren Europäischen Union hat indes längst begonnen. Das Wort vom ,Mythos Europa' macht(-e) in Gazetten die Runde, zumal die Bindewirkung der Europäischen Union während der Eurokrise kräftig auf die Probe gestellt wurde. Die europäische Außenpolitik, das am wenigsten verfestigte Projekt der Europäischen Union, könnte das erste Opfer dieser Fliehkräfte werden, zumal auch die Zusammenlegung sicherheitspolitischer und vor allem militärischer Ressourcen in Europa, trotz vielfältiger Bekundungen und auch Bestrebungen, noch immer zu wünschen übrig lässt. ${ }^{15}$ Auffallend ist, dass jede Projizierung einer machtbesetzten europäischen Außenpolitik aus der Debatte verschwunden zu sein scheint: zu Beginn der letzten Dekade, ungefähr bis zum französischen ,Nein“ zur europäischen Verfassung im Mai 2005, war eine strategische Debatte über eine ,europäische Supermacht' in intellektuellen Zirkeln tonangebend, die in Zusammenhang mit dem Wegbrechen der internationalen Führungsrolle der USA während der zweiten BushAdministration stand. Mit der Aufgabe des ,Supermacht'-Anspruches ist der Europäischen Union aber auch eine potenziell tragfähige gesellschaftliche Utopie abhanden gekommen.

Heute scheint die Europäische Union von einem europäischen Föderalismus und damit auch Elementen außenpolitischer Staatlichkeit weiter entfernt denn je. Indes: auch und gerade eine europäische Außenpolitik bedürfte aggregierter wirtschaftlicher Stärke und einer europäischen Staatlichkeit jenseits der aktuellen Begrifflichkeit von Souveränität. Gemeinsame militärische Macht (,share it or lose it $\left.{ }^{\circ}\right)$ ist für die internationale Schlagkräftigkeit der Europäischen Union ebenso notwendig wie ein starker Euro für die Durchsetzungsfähigkeit ihrer wirtschaftlichen und sozialen Ziele und Werte: die USA haben nicht zuletzt im letzten

13 Vgl. zum Beispiel Werner Abelshauser: Deutschland, Europa und die Welt, in: Frankfurter Allgemeine Zeitung, 9.12.2011.

14 Siehe zum Beispiel die Initiative von Bundesaußenminister Guido Westerwelle, , like-minded‘ EU-Außenminister am 19. März 2012 zum Brainstorming über die Zukunft der Europäischen Union einzuladen.

15 Vgl. Nick Witney: How to Stop the De-Militarization of Europe, ECFR Policy Brief, 2011. 
Jahrhundert auch mit der dominanten Stellung des Dollars im internationalen Währungssystem Außenpolitik gemacht, weil eben wirtschaftliche und strategische Macht und damit Außenpolitik zusammengehören. Es sei an den berühmten Satz von Henry Kissinger an die Europäer erinnert: „Der Dollar ist unsere Währung und euer Problem.“

Politische und ökonomische Macht konsequent zusammen zu denken hieße für Europa, dass der Euro-Raum fiskalisch durchgreifend föderalisiert werden müsste. Stichwörter hierfür wären Eurobonds, ein europäisches Finanzministerium, ein anders geartetes, stärker redistributives EU-Budget, eine europäische Wertschöpfungskette und ein aggregiertes Verständnis des Euro-Raums als einen Wirtschaftsraum anstatt - wie momentan - interne Konkurrenz um Exporte oder Leistungsbilanzen, um überhaupt eine ökonomische Grundlage für eine EU-Außenpolitik zu haben. Außenpolitik und Euro-/Fiskalpolitik müssten stärker zusammengedacht werden: denn es macht zum Beispiel einen Unterschied, ob man China einlädt, sich an der Europäischen Finanzstabilisierungsfazilität (EFSF) zu beteiligen, was im Ergebnis Verlust an Strategiefähigkeit und Unabhängigkeit bedeutet; oder ob man es China aus einer Position der Stärke heraus überlässt, Eurobonds zu kaufen oder nicht, was einen Gewinn an Euro-Liquidität und zudem die Eindämmung von chinesischen , divide et impera‘Strategien mit Blick auf Europa bedeuten würde. ${ }^{16}$ Letzteres würde die Sicherung immaterieller außenpolitischer Werte durch ökonomische Untermauerung der eigenen Machtposition bedeuten; Ersteres deren Ausverkauf. Da es aber nicht danach aussieht, dass eine solche fiskalische Föderalisierung der Europäischen Union trotz Fiskalpakt auf absehbare Zeit gelingt, muss die europäische Außenpolitik leider vorerst zahnlos bleiben - was allerdings den Teufelskreis begründet, dass sie dann nicht überzeugend ist, also keine vernünftige ErgebnisOptimierung stattfindet und sie damit in der Wählergunst immer weiter als, immaterielles Ziel` zurückfallen dürfte.

Das Ineinandergreifen dieser vier Trends dürfte zumindest mittelfristig bedeuten, dass die europäische Außenpolitik und die Konzeptualisierung einer europäischen Rolle in der Welt trotz des Vertrags von Lissabon realistischerweise zumindest vor großen Problemen steht. Fraglich ist zurzeit angesichts der multiplizierten Staatsschulden-, Banken-, Legitimationsund schließlich Vertrauenskrise in der Tat, ob die Europäische Union nicht genau an der Schwelle zu einer Tendenz der schleichenden Desintegration steht, die ihre eigene Dynamik und ihr eigenes Tempo entwickeln könnten und die die Schwerkraft der Europäischen Union in eine andere Richtung beziehungsweise sogar in ihr Auseinanderdriften treiben könnte sollte nicht bald eine nachhaltige Umkehrung dieses Trends beginnen.

16 Vgl. François Godement/Jonas Parello-Plesner/Alice Richard: The Scramble for Europe, ECFR Policy Brief, 2011. 